\title{
Tail dependence of perturbed copulas
}

\author{
Jozef Komorník \\ Faculty of Management, Comenius University, Odbojárov 10, P.O.Box 95, \\ 82005 Bratislava, Slovakia \\ jozef.komornik@fm.uniba.sk \\ Magda Komorníková \\ Faculty of Civil Engineering, Slovak University of Technology, Radlinského 11, \\ 81005 Bratislava, Slovakia \\ magdalena.komornikova@stuba.sk \\ Jana Kalická \\ Faculty of Civil Engineering, Slovak University of Technology, Radlinského 11, \\ 81005 Bratislava, Slovakia \\ jana.kalicka@stuba.sk \\ Cuong Nguyen \\ Faculty of Commerce, PO Box 85084 Lincoln University, Lincoln 7647, \\ Canterbury, New Zealand \\ Cuong.Nguyen@lincoln.ac.nz
}

Received 15 July 2015

Accepted 11 November 2015

In this paper, we extend our investigations of a special class of perturbations of copulas introduced in [7]. Despite a surprising fact that this kind of perturbations does not change the value of tail dependence of the original copulas, their use yielded models with considerably improved fitting qualities.

Keywords: Copula; perturbation of copula; tail dependence; Real Estate Investment Trust (REIT) index; returns of REIT indexes.

2000 Mathematics Subject Classification: 60E99, 62P20, 91B70

\section{Introduction}

Recently, Mesiar et al. (2015) [7] extensively investigated several types of constructions of copulas using their perturbations. One of their constructions yielded a generalization of the FMG (FarlieGumbel-Morgenstern) copula family (see [6])

$$
C_{\alpha}^{F G M}(u, v)=u v+\alpha u(1-u) v(1-v), \quad \alpha \in[-1,1]
$$


in the form

$$
C_{\alpha}(u, v)=C(u, v)+\alpha(u-C(u, v))(v-C(u, v))
$$

for any copula $C=C_{0}$ and for each $\alpha \in[0,1]$. An intuitive desire was expressed for a potential use of perturbations $C_{\alpha}$ for improved modelling of data preliminary modelled by a basic copula $C=C_{0}$. (An illustrative example of fitting copulas to real data was also presented.)

Greater insight into this issue was obtained when a subsequent analysis of tail dependencies (upper and lower) showed that their values remain equal to those for the basic copula. An attempt of a fast publication of this result in Komornik et al. (2014) [5] did not fully succeed because of technical reasons. The problem was that the text of the paper had been (unexpectedly) imprecisely retyped, producing several incorrect (and even senseless) formulations.

Therefore, we briefly present here a precise version of the mentioned results concerning tail dependencies (that also inspired more extensive applications of the perturbed copulas in modelling real data). Following our earlier investigations of more classical financial instruments (that have been also treated by many other authors), we focus at less usual objects of investigations, so called REIT (Real Estate Investment Trust) indexes. We were curious if their behaviour has been influenced by the GFC (similarly as was the case of more standard capital market instruments). Eventually, following a suggestion of a referee, we apply our modelling approach to data from Bhatti and Nguyen (2012) [2].

The paper is organized as follows. The second section is devoted to a brief overview of the bivariate copulas and their perturbations. In the third section the utilized methodology of copula fitting to two-dimensional time series is presented. The fourth section presents applications to modelling of selected time series of REIT indexes. Finally, some conclusions are presented.

\section{Bivariate copulas and their perturbations}

In our modelling applications, we will make use of some Archimedean copulas, corresponding survival copulas and their convex combinations. Based on the referees suggestions, details of definitions of those copulas, the meaning of their parameters and formulas for their coefficients of tail dependence $\lambda_{L}$ and $\lambda_{U}$ are skipped (since they can be found e.g. in Nelsen (2006) [8] and partially also in Nguyen et al. (2014) [3]).

For any copula $C$ we denote its survival copula by $\widehat{C}$ and use the obvious relations

$$
\lambda_{U}(\widehat{C})=\lambda_{L}(C)
$$

and

$$
\lambda_{L}(\widehat{C})=\lambda_{U}(C) .
$$

For selected one parametric subclasses of Archimedean copulas with the standard meaning of their parameters $\theta$ we use the following notations:

- Clayton (strict) $C_{\theta}^{C l}$ for $\theta \in(0, \infty)$,

- Gumbel $C_{\theta}^{G}$ for $\theta \in[1, \infty)$,

- Joe (BB5) $C_{\theta}^{J}$ for $\theta \in[1, \infty)$.

Let us recall that for Clayton type copulas the coefficients $\lambda_{L}$ equal to 0 , while for Gumbel and Joe type copulas the coefficients $\lambda_{U}$ equal to 0 . Convenient forms of models with equal positive values of $\lambda_{L}$ and $\lambda_{U}$ are corresponding weighted averages $0.5(C+\widehat{C})$ of the above three types of copulas. 
One of the main results of Mesiar et al. (2015) [7] is that for any copula $C$ and $\alpha \in[0,1]$, the relation (1.1) defines a copula. Because of the mentioned problems with Komornik et al. (2014) [5], we repeat the reason for equivalent values of coefficients $\lambda_{L}$ and $\lambda_{U}$ for any copula $C$ and corresponding copulas $C_{\alpha}$ given by (1.1). It is obvious that an equivalent definition of $\lambda_{L}(C)$ can be obtained via the diagonal section function given by

$$
\delta_{C}:[0,1] \rightarrow[0,1], \delta_{C}(u)=C(u, u)
$$

as

$$
\lambda_{L}(C)=\lim _{u \rightarrow 0^{+}} \delta_{C}(u)=\delta_{C}^{\prime}(u) .
$$

For any $\alpha \in[0,1]$ and $C_{\alpha}$ given by (1.1) we have

$$
\delta_{C}(u) \leq \delta_{C_{\alpha}}(u)=\delta_{C}(u)+\alpha\left(u-\delta_{C}(u)\right)^{2} \leq \delta_{C}(u)+\alpha u^{2} .
$$

Thus

$$
\lambda_{L}(C)=\lambda_{L}\left(C_{\alpha}\right)
$$

It is easy to note that

$$
\lambda_{U}(C)=\lambda_{L}(\widehat{C})=\lambda_{L}\left(\widehat{C}_{\alpha}\right)=\lambda_{U}\left(C_{\alpha}\right) .
$$

\section{Remark 2.1}

We can conclude that the perturbations $C_{\alpha}$ given by (1.1) do not change the values of tail dependence coefficients of the original copulas $C$. So we can consider these perturbations (in some sense) as minor even for values of the parameter $\alpha$ that are not close to 0 .

\section{Fitting of copulas}

In practical fitting of the data we have utilized the maximum pseudolikelihood method (MPL) of parameter estimation with initial parameters estimates received by the minimalization of the mean square distance to the empirical copula $C_{n}$ presented e.g. in [4]

$$
C_{n}(u, v)=\frac{1}{n} \sum_{i=1}^{n} \mathbf{1}\left(\frac{R_{i}}{n+1} \leq u, \frac{S_{i}}{n+1} \leq v\right)
$$

where $n$ is the sample size, $R_{i}$ stands for the rank of $X_{i}$ among $X_{1}, \ldots, X_{n}, S_{i}$ stands for the rank of $Y_{i}$ among $Y_{1}, \ldots, Y_{n}$ and $\mathbf{1}(\Omega)$ denoting the indicator function of set $\Omega$. It requires that the copula $C_{\theta}(u, v)$ is absolutely continuous with density $c_{\theta}(u, v)=\frac{\partial^{2}}{\partial u \partial v} C_{\theta}(u, v)$. This method (described e.g. in [4]) involves maximizing a rank-based log-likelihood of the form

$$
L(\theta)=\sum_{i=1}^{n} \ln \left(c_{\theta}\left(\frac{R_{i}}{n+1} ; \frac{S_{i}}{n+1}\right)\right)
$$

where $\theta$ is vector of parameters in the model. Note that arguments $\frac{R_{i}}{n+1}, \frac{S_{i}}{n+1}$ equal to the corresponding values of the empirical marginal distributional functions of random variables $X$ and $Y$. 
For selecting the optimal models we applied the Kolmogorov - Smirnov - Anderson - Darling (KSAD, for which we use the abbreviation $A D$ ) test statistic defined e.g. in [1]

$$
A D(\theta)=\sqrt{n} \max \left|\frac{C_{n}\left(\frac{R_{i}}{n+1}, \frac{S_{i}}{n+1}\right)-C_{\theta}\left(\frac{R_{i}}{n+1}, \frac{S_{i}}{n+1}\right)}{C_{\theta}\left(\frac{R_{i}}{n+1}, \frac{S_{i}}{n+1}\right) *\left(1-C_{\theta}\left(\frac{R_{i}}{n+1}, \frac{S_{i}}{n+1}\right)\right)}\right|
$$

for which we also constructed a GoF simulation based test, when comparing models with their submodels and different families of models.

\section{Application to real data modelling}

A REIT (Real Estate Investment Trust) is a company that mainly owns, and in most cases, operates income-producing real estate such as apartments, shopping centres, offices, hotels and warehouses. Some REITs also engage in financing real estate. The shares of many REITs are traded on major stock exchanges.

REIT Index Series is designed to present investors with a comprehensive family of REIT performance indexes that spans the commercial real estate space across the economy of the country. The index series provides investors with exposure to all investment and property sectors. In addition, the more narrowly focused property sector and sub-sector indexes provide the facility to concentrate commercial real estate exposure in more selected markets.

We have investigated the relations between 4 selected countries' (USA, Australia, Japan and UK) daily returns of the REIT (Real Estate Investment Trust) indexes in different time periods, determined by the recent global financial markets crises (July 1, 2008 - April 30, 2009) that can be also clearly identified from the following Figure 1, presenting the parallel development of the considered REIT indexes.
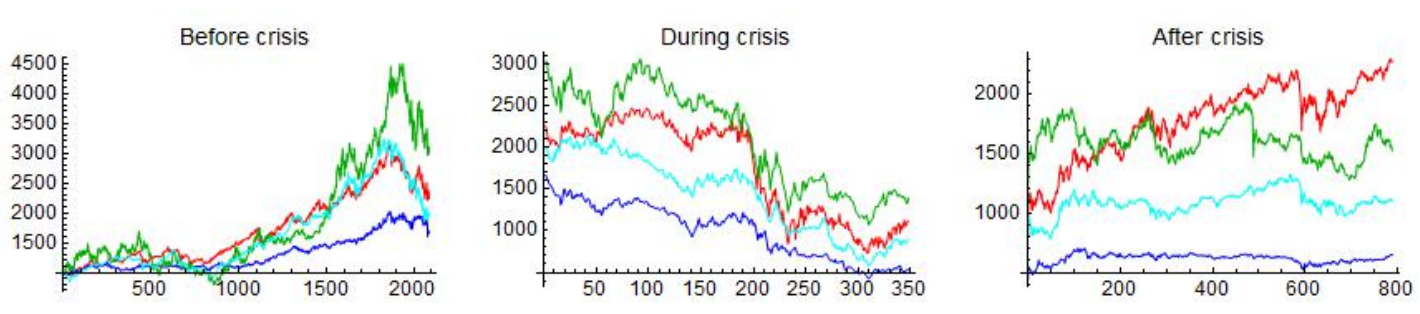

Fig. 1. Real Estate Investment Trust indexes in different time periods $(\mathrm{USA}=$ red, Australia = blue, Japan = green, UK= cyan)

We have performed filtering of the returns of all individual REIT indexes (in order to avoid a possible violation of the i.i.d. property) by ARMA-GARCH models (separately for the individual considered time sub-periods).

For all three time sub-periods and all couples of (filtered) returns of the REIT indexes we have performed the non-parametric correlation analyses based on the Kendall coefficients (see Table 1, Table 2 and Table 3). We have observed that the values of the correlation coefficients have dropped substantially between the first and the second considered time sub-periods and even more dramatically for the third sub-period. These changes are illustrated in the scatter plots (see Figure 2 Figure 7). 
Table 1. The values of the Kendall's correlation coefficient $\tau$ for the pre-crisis period

\begin{tabular}{lllll}
\hline before crisis & USA & Australia & Japan & UK \\
\hline USA & 1 & 0.994 & 0.731 & 0.737 \\
Australia & 0.994 & 1 & 0.727 & 0.738 \\
Japan & 0.731 & 0.727 & 1 & 0.609 \\
UK & 0.737 & 0.738 & 0.609 & 1 \\
\hline
\end{tabular}

Table 2. The values of the Kendall's correlation coefficient $\tau$ for the crisis period

\begin{tabular}{lllll}
\hline during crisis & USA & Australia & Japan & UK \\
\hline USA & 1 & 0.301 & 0.267 & 0.306 \\
Australia & 0.301 & 1 & 0.535 & 0.397 \\
Japan & 0.267 & 0.535 & 1 & 0.378 \\
UK & 0.306 & 0.397 & 0.378 & 1 \\
\hline
\end{tabular}

Table 3. The values of the Kendall's correlation coefficient $\tau$ for the post-crisis period

\begin{tabular}{lllll}
\hline after crisis & USA & Australia & Japan & UK \\
\hline USA & 1 & 0.111 & 0.061 & 0.221 \\
Australia & 0.111 & 1 & 0.222 & 0.087 \\
Japan & 0.061 & 0.222 & 1 & 0.073 \\
UK & 0.221 & 0.087 & 0.073 & 1 \\
\hline
\end{tabular}
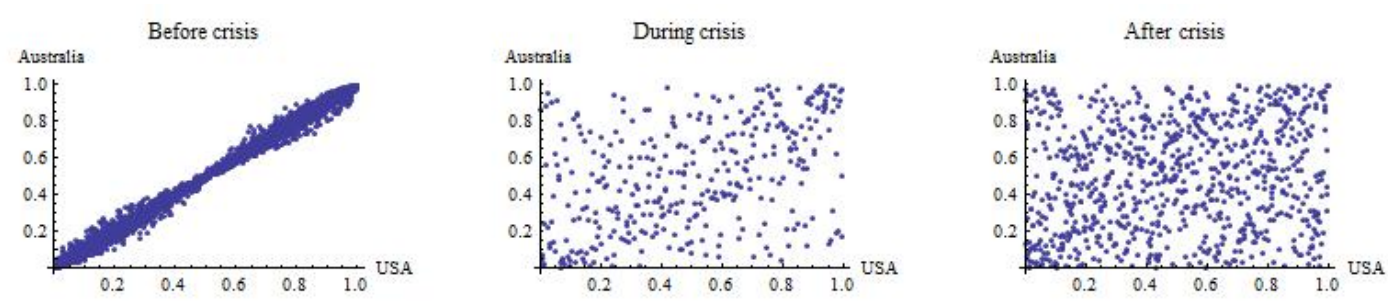

Fig. 2. USA \& Australia

We have applied the fitting by copulas to the residuals of ARMA-GARCH filters. We considered models from strict Archimedean copulas (Joe $C^{J}$, Clayton $C^{C l}$ and Gumbel $C^{G}$ ) families and their mixtures with corresponding survival copulas $\hat{C}$ as well as their perturbations given by (1.1).

To estimate parameters for each type of models we have used the maximum pseudo-likelihood method. For selecting the optimal models we have applied the Kolmogorov - Smirnov - Anderson - Darling (for which we have used the abbreviation $A D$ ) test statistic (3.1). For all of them, the 

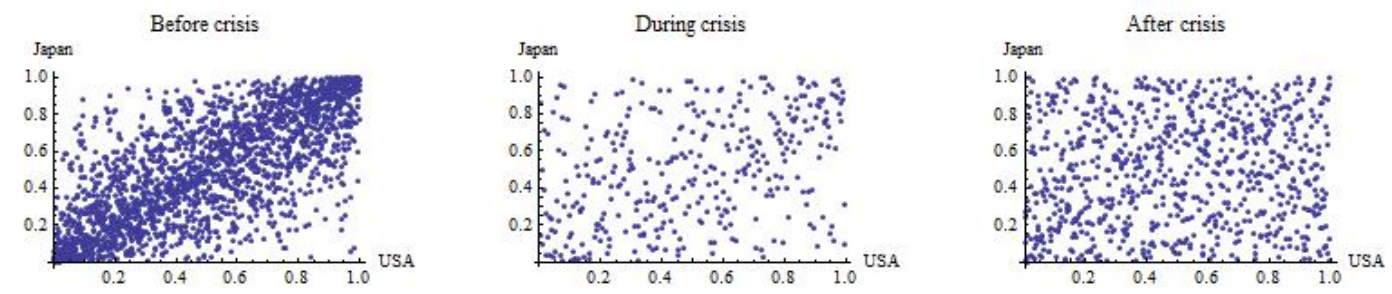

Fig. 3. USA \& Japan
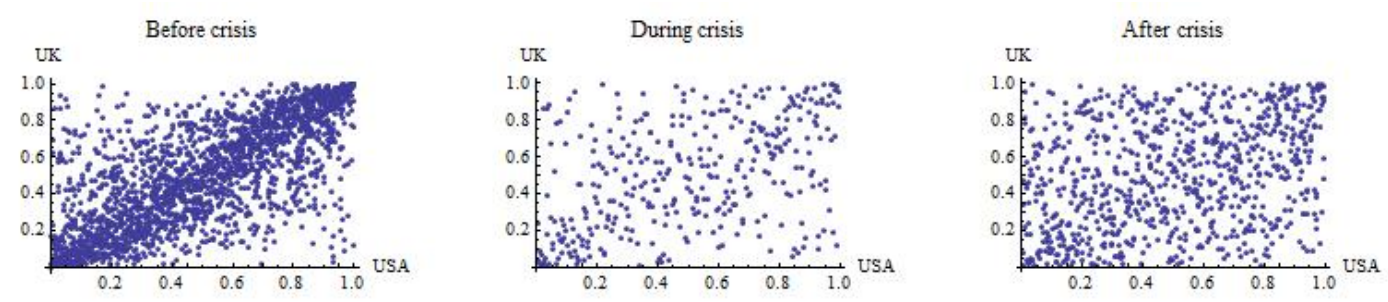

Fig. 4. USA \& U.K.
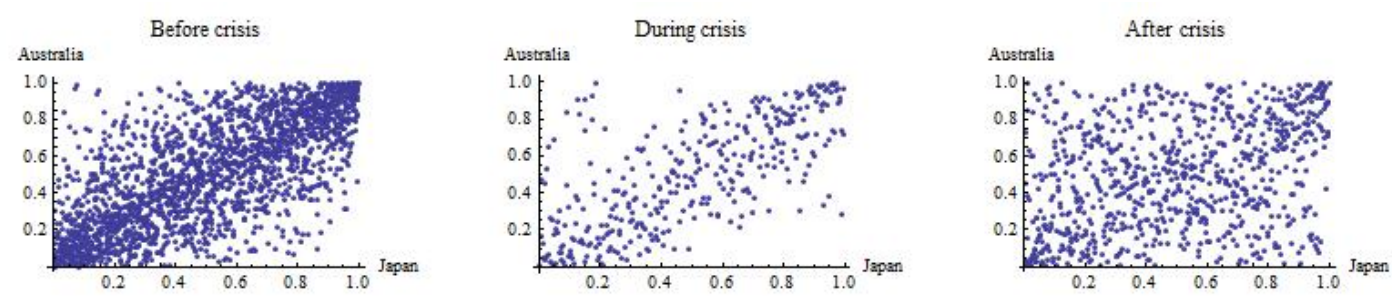

Fig. 5. Japan \& Australia
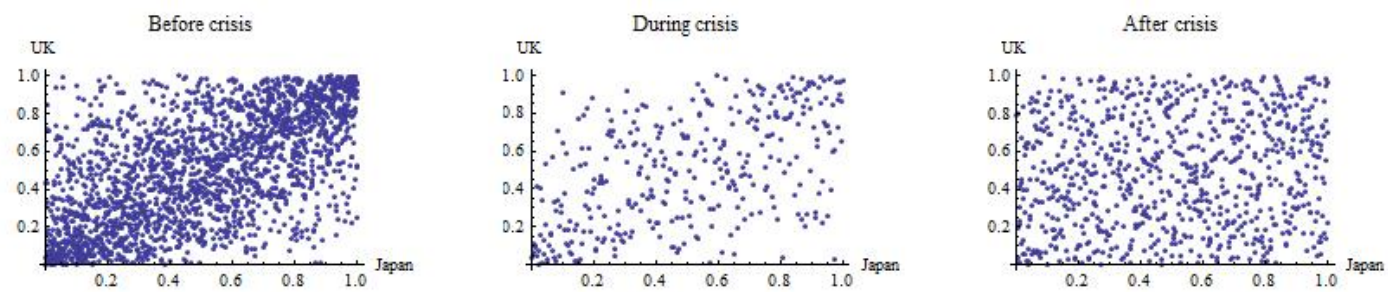

Fig. 6. Japan \& U.K.

simulation based GoF test yielded p-value $>0.1$. The overview of best types of copulas for all couples and all time sub-periods of the filtered returns of REIT indexes is found in Table 4, Table 5 and Table 6.

We can observe that most values of the coefficients of tail dependencies for the optimal copula models (with few stagnating exceptions) change between individual considered time periods in a 

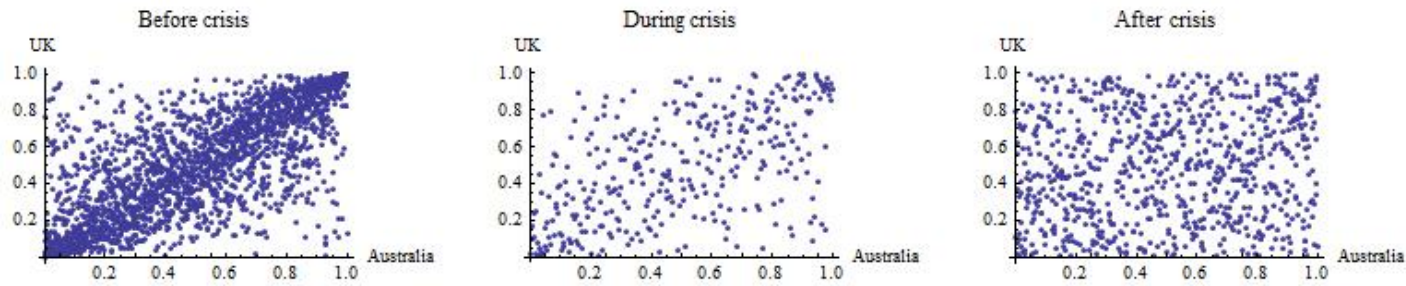

Fig. 7. Australia \& U.K.

Table 4. The overview of the best types of copulas for all couples of the (filtered) returns of REIT indexes before crisis

\begin{tabular}{llllll}
\hline Couple & the best types of copula $C$ & $\alpha$ & $\theta$ & $\lambda_{L}$ & $\lambda_{U}$ \\
\hline USA \& Japan & $0.5 *\left(C^{C l}+\widehat{C}^{C l}\right)$ & 0.98 & 1.71 & 0.33 & 0.33 \\
USA \& Australia & $0.5 *\left(C^{G}+\widehat{C}^{G}\right)$ & 0.97 & 6.64 & 0.44 & 0.44 \\
USA \& U.K. & $0.5 *\left(C^{G}+\widehat{C}^{G}\right)$ & 0.07 & 2.32 & 0.33 & 0.33 \\
Japan \& Australia & $0.5 *\left(C^{G}+\widehat{C}^{G}\right)$ & 0.89 & 1.91 & 0.28 & 0.28 \\
Japan \& U.K. & $0.5 *\left(C^{G}+\widehat{C}^{G}\right)$ & 0.55 & 1.64 & 0.24 & 0.24 \\
U.K \& Australia & $0.5 *\left(C^{G}+\widehat{C}^{G}\right)$ & 0.02 & 2.33 & 0.33 & 0.33 \\
\hline
\end{tabular}

Table 5. The overview of the best types of copulas for all couples of the (filtered) returns of REIT indexes during crisis

\begin{tabular}{llllll}
\hline Couple & the best types of copula $C$ & $\alpha$ & $\theta$ & $\lambda_{L}$ & $\lambda_{U}$ \\
\hline USA \& Japan & $0.5 *\left(C^{G}+\widehat{C}^{G}\right)$ & 0.46 & 1.24 & 0.12 & 0.12 \\
USA \& Australia & $0.5 *\left(C^{G}+\widehat{C}^{G}\right)$ & 0.05 & 1.44 & 0.19 & 0.19 \\
USA \& U.K. & $C^{C l}$ & 0.41 & 0.65 & 0.34 & 0.00 \\
Japan \& Australia & $0.5 *\left(C^{G}+\widehat{C}^{G}\right)$ & 0.44 & 2.01 & 0.29 & 0.29 \\
Japan \& U.K. & $0.5 *\left(C^{G}+\widehat{C}^{G}\right)$ & 0.48 & 1.49 & 0.21 & 0.21 \\
U.K \& Australia & $0.5 *\left(C^{G}+\widehat{C}^{G}\right)$ & 0.17 & 1.62 & 0.23 & 0.23 \\
\hline
\end{tabular}

Table 6. The overview of the best types of copulas for all couples of the (filtered) returns of REIT indexes after crisis

\begin{tabular}{llllll}
\hline Couple & the best types of copula $C$ & $\alpha$ & $\theta$ & $\lambda_{L}$ & $\lambda_{U}$ \\
\hline USA \& Japan & $0.5 *\left(C^{J}+\widehat{C}^{J}\right)$ & 0.23 & 1.13 & 0.07 & 0.07 \\
USA \& Australia & $C^{C l}$ & 0.10 & 0.19 & 0.03 & 0.00 \\
USA \& U.K. & $0.5 *\left(C^{G}+\widehat{C}^{G}\right)$ & 0.06 & 1.29 & 0.14 & 0.14 \\
Japan \& Australia & $0.5 *\left(C^{J}+\widehat{C}^{J}\right)$ & 0.03 & 1.51 & 0.21 & 0.21 \\
Japan \& U.K. & $C^{C l}$ & 0.02 & 0.15 & 0.01 & 0.00 \\
U.K \& Australia & $C^{C l}$ & 0.24 & 0.10 & 0.001 & 0.00 \\
\hline
\end{tabular}

similar way as the values of the Kendall correlation coefficients. They also have dropped between the first and the second period and continued to fall between the second and the third period. 
Subsequently, we applied the above modelling procedure for the data from Bhatti and Nguyen (2012) [2] for the couple of the Australian Stock Index and Australian Futures Index that span for the period before the GFC. The optimal model was again provided by a perturbed copula of the form (1.1) with $C=0.5\left(C^{G}+\widehat{C}^{G}\right)$ and $\alpha=0.77, \theta=3.11, \lambda_{L}=\lambda_{U}=0.37$.

\section{Concluding remarks}

In the theoretical part of the paper, we derived an important result for the special type of perturbed copulas, where the perturbations do not change the values of tail dependencies. In the practical part, we observed strongly decreasing trends (between the subsequent considered time periods) for the values of the Kendall correlation coefficients as well as the values of tail dependence coefficients for the optimal copula models (for most considered couples of filtered returns of REIT indexes).

Subsequently our modelling approach was successfully verified for a completely different financial data from an earlier publication of Bhatti and Nguyen (2012) [2]

Despite the theoretical fact that the considered class of perturbations does not change the tail dependence coefficients of the considered copulas, their use yielded a considerable improvement (with respect to the $\mathrm{AD}$ criterion).

\section{Acknowledgments}

The support of the grants APVV-0236-12 and APVV-14-0013 is kindly announced.

\section{References}

[1] D. Berg and H. Bakken, Copula Goodness-of-fit Tests: A Comparative Study, Working paper (University of Oslo and Norwegian Computing Center, 2006)

[2] M.I. Bhatti and C.C. Nguyen, Diversification evidence from international equity markets using extreme valeus and stochastic copulas, Jounal of International Financial Markets, Institutions \& Money 22 (2012) 622-646

[3] C. Nguyen, M.I. Bhatti and A. Hayat, Volatility linkages in the spot and futures market in Australia: a copula approach, Qual. Quant 48 (2014) 2589-2603

[4] C. Genest and A.C. Favre, Everything you always wanted to know about copula modeling but were afraid to ask, Journal of Hydrologic Engineering 12 (2007) 347-368

[5] J. Komorník, M. Komorníková and J. Kalická, Perturbed Copulas and Their Tail Dependencies. Global Journal For Research Analysis (GJRA)3/12 (2014) 69-70

[6] P. Kumar, Probability Distributions and Estimation of Ali-Mikhail-Haq Copula, Applied Mathematical Sciences 4/14 (2010) 657-666

[7] R. Mesiar, M. Komorníková and J. Komorník, Perturbation of bivariate copula, Fuzzy Sets and System 268 (2015) 127-140

[8] R.B. Nelsen, An introduction to copulas, 2nd edn. Springer Series in Statistics, (Springer-Verlag, New York, 2007) 